\title{
Total Edge Irregularity Strength of the Disjoint Union of Helm Graphs
}

\author{
Muhammad Kamran Siddiqui ${ }^{1}, \operatorname{Nurdin}^{2}$ \& Edy Tri Baskoro ${ }^{3}$ \\ ${ }^{1}$ Abdus Salam School of Mathematical Sciences, \\ 68-B New Muslim Town, GC University, Lahore, Pakistan \\ ${ }^{2}$ Mathematics Department, Faculty of Mathematics, and Natural Sciences, Hasanuddin \\ University, Jalan Perintis Kemerdekaan, Km.10 Tamalanrea, Makassar, Indonesia \\ ${ }^{3}$ Combinatorial Mathematics Research Group, Faculty of Mathematics and Natural \\ Sciences, Institut Teknologi Bandung, Jalan Ganesa No. 10. Bandung 40132, Indonesia \\ Email: kamransiddiqui75@gmail.com
}

\begin{abstract}
The total edge irregular k-labeling of a graph $G=(V, E)$ is the labeling of vertices and edges of $G$ in such a way that for any different edges their weights are distinct. The total edge irregularity strength, tes $(G)$, is defined as the minimum $k$ for which $G$ has a total edge irregular $k$-labeling. In this paper, we consider the total edge irregularity strength of the disjoint union of $\mathrm{m}$ special types of helm graphs.
\end{abstract}

Keywords: disjoint union; edge irregular total labeling; helm graph; irregularity strength; total edge irregularity strength.

\section{$1 \quad$ Introduction}

In this paper, we consider a graph $G$ as a finite graph (without loop and multiple edges) with the vertex-set $V$ and the edge-set $E$. In [1], Baca, Jendrol, Miller and Ryan introduced the notion of the total edge irregular $k$-labeling of a graph $G=(V, E$ namely the labeling $\psi: V \cup E \rightarrow\{1,2, \ldots, k\}$ such that all edge weights are different. The weight $w t(u v)$ of an edge $u v$ is defined as $w t_{\psi}(u v)=\psi(u)+\psi(u v)+\psi(v)$. The total edge irregularity strength of $G$, denoted by $\operatorname{tes}(G)$, is the smallest $k$ for which $G$ has a total edge irregular $k$ labeling.

The basic idea of the total edge irregularity strength came from irregular assignments and the irregularity strength of graphs introduced by Chartrand, Jacobson, Lehel, Oellermann, Ruiz and Saba [2]. An irregular assignment is a $k$-labeling of the edges such that the sum of the labels of edges incident to a vertex is different for all the vertices of $G$. The smallest integer $k$ for which $G$ has an irregular assignment is called the irregularity strength of $G$, and is denoted by $s(G)$.

Received October $25^{\text {th }}, 2012$, Revised May $30^{\text {th }}, 2013$, Accepted for publication June $4^{\text {th }}, 2013$.

Copyright () 2013 Published by ITB Journal Publisher,ISSN: 2337-5760, DOI: 10.5614/j.math.fund.sci.2013.45.2.6 
It is not an easy task to compute the irregularity strength of graphs with simple structures, see [3-6]. Karonski, Luczak and Thomason [7] conjectured that the edges of every connected graph of order at least 3 can be assigned labels from $\{1,2,3\}$ such that for all pairs of adjacent vertices the sums of the labels of the incident edges are distinct. Baca, Jendrol, Miller and Ryan [1] gave a lower bound on the total edge irregularity strength of a graph:

$$
\operatorname{tes}(G) \geq \max \left\{\left\lceil\frac{|E(G)|+2}{3}\right\rceil,\left\lceil\frac{\Delta(G)+1}{2}\right\rceil\right\}
$$

where $\Delta(G)$ is the maximum degree of $G$. The authors of [1] determined the exact values of the total edge irregularity strength for paths, cycles, stars, wheels and friendship graphs. Recently, Ivanco and Jendrol [8] posed the following conjecture:

Conjecture 1. Let $G$ be an arbitrary graph different from $K \_5$. Then

$$
\operatorname{tes}(G)=\max \left\{\left\lceil\frac{|E(G)|+2}{3}\right\rceil,\left\lceil\frac{\Delta(G)+1}{2}\right\rceil\right\}
$$

Conjecture 1 has been verified for all trees in [8], for complete graphs and complete bipartite graphs in [9] and [10], for the Cartesian product of two paths $P_{n} \square P_{m}$ in [11], for the corona product of a path with certain graphs in [12], for large dense graphs with $\frac{|E(G)|+2}{3} \leq \frac{\Delta(G)+1}{2}$ in [13], for hexagonal grids in [14], for the zigzag graph [15], for the categorical product of two paths $P_{n} \times P_{m}$ [16], for the categorical product of a cycle and a path $C_{n} \times P_{m}$ in [17,18], for a subdivision of stars in [19], for the categorical product of two cycles in [20], and for the strong product of two paths in [21].

Motivated by [22], we investigated the total edge irregularity strength of the disjoint union of helm graphs. A helm graph $H_{n}$ is obtained from a wheel on $n+1$ vertices by adding a pendant edge to every vertex of its cycle $C_{n}$. In this study, we determined the total edge irregularity strength of the disjoint union of $m$ copies of a certain helm graph. We also determined the total edge irregularity strength of the disjoint union of non-isomorphic helm graphs.

This paper adds further support to Conjecture 1 by demonstrating that the disjoint union of helm graphs has a total edge irregularity strength equal to 


$$
\left\lceil\frac{\left|E\left(\bigcup_{j=1}^{m} H_{n+j}\right)\right|+2}{3}\right\rceil .
$$

\section{$2 \quad$ Main Results}

First, we determine the total edge irregularity strength of a disjoint union $m H_{n}$ of $m$ copies of a helm graph $H_{n}$. Let

$$
\begin{aligned}
& V\left(H_{n}\right)=\left\{c^{j}, x_{i}^{j}, y_{i}^{j} ; 1 \leq i \leq n, 1 \leq j \leq m\right\} \\
& E\left(H_{n}\right)=\left\{c^{j} x_{i}^{j}, x_{i}^{j} y_{i}^{j}, x_{i}^{j} x_{i+1}^{j} ; 1 \leq i \leq n, 1 \leq j \leq m\right\}
\end{aligned}
$$

Moreover, the subscript $n+1$ is replaced by 1 .

Lemma 1. For $n \geq 3$ tes $\left(2 H_{n}\right)=2 n+1$.

Proof. From (1) it follows that tes $\left(2 H_{n}\right) \geq 2 n+1$. Now the existence of an optimal labeling $\varphi_{1}$ proves the converse inequality for $1 \leq i \leq n$ as follows:

$$
\begin{aligned}
& \varphi_{1}\left(x_{i}^{1}\right)=\varphi_{1}\left(y_{i}^{1}\right)=1, \varphi_{1}\left(c^{1}\right)=\varphi_{1}\left(c^{2}\right)=2 n+1, \\
& \varphi_{1}\left(c^{1} x_{i}^{1}\right)=\varphi_{1}\left(x_{i}^{2} x_{i+1}^{2}\right)=\varphi_{1}\left(x_{i}^{1} y_{i}^{1}\right)=\varphi_{1}\left(x_{i}^{2} y_{i}^{2}\right)=i, \\
& \varphi_{1}\left(x_{i}^{2}\right)=2 n+1, \quad \varphi_{1}\left(y_{i}^{2}\right)=n+1, \quad \varphi_{1}\left(c^{2} x_{i}^{2}\right)=\varphi_{1}\left(x_{i}^{1} x_{i+1}^{1}\right)=n+i .
\end{aligned}
$$

It is easy to see that the weights of the edges are pair-wise distinct. This concludes the proof.

Theorem 1. Let $m, n \geq 3$ be two integers. Then, the total edge irregularity strength of a disjoint union $m H_{n}$ of $m$ copies of a helm graph $H_{n}$ is $m n+1$.

Proof. As $\left|\mathrm{E}\left(m H_{n}\right)\right|=3 m n$ then (1) implies that tes $\left(H_{n}\right) \geq m n+1$. Let $k=$ $m n+1$. To prove the converse inequality, we define the total edge irregular $k$ labeling $\psi_{1}$ for $1 \leq i \leq n$ and $1 \leq j \leq m$ as follows: 


$$
\psi_{1}\left(c^{j}\right)=\psi_{1}\left(x_{i}^{j}\right)=\psi_{1}\left(y_{i}^{j}\right)=\min \left\{\left\lfloor\frac{3 n(j-1)+2}{2}\right\rfloor, k\right\}
$$

Case I: For $1 \leq j \leq m$ such that $\left\lfloor\frac{3 n(j-1)+2}{2}\right\rfloor<k$

(i) When $n$ is even,

$$
\psi_{1}\left(x_{i}^{j} y_{i}^{j}\right)=i, \psi_{1}\left(x_{i}^{j} x_{i+1}^{j}\right)=n+i, \psi_{1}\left(c^{j} x_{i}^{j}\right)=2 n+i,
$$

(ii) When $n$ is odd,

(a) If $j$ is odd, then the edges $c^{j} x_{i}^{j}, x_{i}^{j} y_{i}^{j}$ and $x_{i}^{j} x_{i+1}^{j}$ receive the same labels as in Case I (i)

(b) If $j$ is even,

$$
\psi_{1}\left(x_{i}^{j} y_{i}^{j}\right)=1+i, \quad \psi_{1}\left(x_{i}^{j} x_{i+1}^{j}\right)=n+1+i, \quad \psi_{1}\left(c^{j} x_{i}^{j}\right)=2 n+1+i,
$$

Case II: For $1 \leq j \leq m$ such that $\left\lfloor\frac{3 n(j-1)+2}{2}\right\rfloor \geq k$

Let

$$
\begin{aligned}
& w=\min \left\{j ; 1 \leq j \leq m \text { such that }\left\lfloor\frac{3 n(j-1)+2}{2}\right\rfloor \geq k\right\} \\
& l=\max \left\{t_{j} ; 1 \leq j \leq m \text { such that }\left\lfloor\frac{3 n(j-1)+2}{2}\right\rfloor<k\right\} \\
& t_{j}=\min \left\{\left\lfloor\frac{3 n(j-1)+2}{2}\right\rfloor, \mathrm{k}\right\} \text { for } 1 \leq j \leq m \text { such that }\left\lfloor\frac{3 n(j-1)+2}{2}\right\rfloor<k
\end{aligned}
$$

(i) When $n$ is even,

$$
\begin{aligned}
& \psi_{1}\left(x_{i}^{j} y_{i}^{j}\right)= \begin{cases}3 n+2(l-k)+i, & \text { if } \quad j=w \\
3 n+2(l-k)+i+(j-w) 3 n, & \text { if } \quad w+1 \leq j \leq m\end{cases} \\
& \psi_{1}\left(x_{i}^{j} x_{i+1}^{j}\right)= \begin{cases}4 n+2(l-k)+i, & \text { if } \quad j=w \\
4 n+2(l-k)+i+(j-w) 3 n, & \text { if } \quad w+1 \leq j \leq m\end{cases} \\
& \psi_{1}\left(c^{j} y_{i}^{j}\right)= \begin{cases}5 n+2(l-k)+i, & \text { if } \quad j=w \\
5 n+2(l-k)+i+(j-w) 3 n, & \text { if } \quad w+1 \leq j \leq m\end{cases}
\end{aligned}
$$

(ii) When $n$ is odd,

(a) If $w$ is odd

$$
\psi_{1}\left(x_{i}^{j} y_{i}^{j}\right)=\left\{\begin{array}{llc}
3 n+2(l-k)+1+i, & \text { if } & j=w \\
3 n+2(l-k)+i+1+(j-w) 3 n, & \text { if } & w+1 \leq j \leq m
\end{array}\right.
$$




$$
\begin{aligned}
& \psi_{1}\left(x_{i}^{j} x_{i+1}^{j}\right)= \begin{cases}4 n+2(l-k)+1+i, & \text { if } \quad j=w \\
4 n+2(l-k)+i+1+(j-w) 3 n, & \text { if } \quad w+1 \leq j \leq m\end{cases} \\
& \psi_{1}\left(c^{j} y_{i}^{j}\right)= \begin{cases}5 n+2(l-k)+1+i, & \text { if } \quad j=w \\
5 n+2(l-k)+i+1+(j-w) 3 n, & \text { if } \quad w+1 \leq j \leq m\end{cases}
\end{aligned}
$$

(b) If $w$ is even, then the edges $c^{j} x_{i}^{j}, x_{i}^{j} y_{i}^{j}$ and $x_{i}^{j} x_{i+1}^{j}$ receive the same labels as in Case II (i)

Under the labeling $\psi_{1}$ the total weights of the edges are described as follows:

(i) The edges $x_{i}^{j} y_{i}^{j}$ for $1 \leq i \leq n, 1 \leq j \leq m$ receive consecutive integers from the interval $[3 n(j-1)+3,3 n(j-1)+n+2]$,

(ii) The edges $x_{i}^{j} x_{i+1}^{j}$ for $1 \leq i \leq n, 1 \leq j \leq m$ receive consecutive integers from the interval $[3 n(j-1)+n+3,3 n(j-1)+2 n+2]$,

(iii) The edges $c^{j} x_{i}^{j}$ for $1 \leq i \leq n, 1 \leq j \leq m$ receive consecutive integers from the interval $[3 n(j-1)+2 n+3,3 n(j-1)+3 n+2]$.

It is not difficult to see that all vertex and edge labels are at most $k$ and the edgeweights of the edges $c^{j} x_{i}^{j}, x_{i}^{j} y_{i}^{j}$ and $x_{i}^{j} x_{i+1}^{j}$ are pairwise distinct. Thus, the resulting labeling is a total edge irregular $k$-labeling. This concludes the proof.

For $m, n \geq 2$, let us consider the disjoint union of $\mathrm{m}$ non-isomorphic helm graphs: $H_{n+1}, H_{n+2}, H_{n+3}, \ldots, H_{n+m}$, where

$$
V\left(\cup_{j=1}^{m} H_{n+j}\right)=\left\{c^{j}, x_{i}^{j}, y_{i}^{j} ; 1 \leq i \leq n+j, 1 \leq j \leq m\right\}
$$

is the corresponding vertex set and

$$
E\left(\cup_{j=1}^{m} H_{n+j}\right)=\left\{c^{j} x_{i}^{j}, x_{i}^{j} y_{i}^{j}, x_{i}^{j} x_{i+1}^{j} ; 1 \leq i \leq n+j, 1 \leq j \leq m\right\}
$$

is the corresponding edge set. Note that the subscript $n+j+1$ is replaced by 1 .

Now, we determine the exact value of the total edge irregularity strength of the graph $\cup_{j=1}^{m} H_{n+j}$. 
Theorem 1. Let $m, n \geq 2$ be two integers and $G \cong \bigcup_{j=1}^{m} H_{n+j}$. Then $\operatorname{tes}(G)=m n+1+\frac{m(m+1)}{2}$.

Proof. As $\left|\mathrm{E}\left(\bigcup_{j=1}^{m} H_{n+j}\right)\right|=3 \sum_{j=1}^{m}(n+j)$ then from (1) it follows that tes $(G) \geq m n+1+\frac{m(m+1)}{2}$. Let $k=m n+1+\frac{m(m+1)}{2}$. To prove the converse inequality, we define the total edge irregular $k$-labeling $\psi_{2}$ for $1 \leq i \leq n+j$ and $1 \leq j \leq m$ as follows.

For $1 \leq i \leq n+1$

$$
\begin{gathered}
\psi_{2}\left(c^{1}\right)=\psi_{2}\left(x_{i}^{1}\right)=\psi_{2}\left(y_{i}^{1}\right)=1, \\
\psi_{2}\left(x_{i}^{1} y_{i}^{1}\right)=i, \quad \psi_{2}\left(x_{i}^{1} x_{i+1}^{1}\right)=n+1+i, \quad \psi_{2}\left(c^{1} x_{i}^{1}\right)=2 n+2+i,
\end{gathered}
$$

For $1 \leq i \leq n+j$

$\psi_{2}\left(c^{j}\right)=\psi_{2}\left(x_{i}^{j}\right)=\psi_{2}\left(y_{i}^{j}\right)=\min \left\{\left\lfloor\frac{3 \sum_{s=1}^{j-1}(n+s+2)}{2}\right\rfloor, k\right\}$ for $2 \leq j \leq m$.

- For $2 \leq j \leq m$ such that $\left\lfloor\frac{3 \sum_{s=1}^{j-1}(n+s)+2}{2}\right\rfloor<\mathrm{k}$

(i) When $\sum_{s=1}^{j-1}(n+s) \equiv 0(\bmod 2)$

$$
\psi_{2}\left(x_{i}^{j} y_{i}^{j}\right)=i, \quad \psi_{2}\left(x_{i}^{j} x_{i+1}^{j}\right)=n+j+i, \quad \psi_{2}\left(c^{j} x_{i}^{j}\right)=2 n+2 j+i,
$$

(ii) When $\sum_{s=1}^{j-1}(n+s) \equiv 1(\bmod 2)$

$$
\psi_{2}\left(x_{i}^{j} y_{i}^{j}\right)=1+i, \quad \psi_{2}\left(x_{i}^{j} x_{i+1}^{j}\right)=1+n+j+i, \quad \psi_{2}\left(c^{j} x_{i}^{j}\right)=1+2 n+2 j+i,
$$


- For $2 \leq j \leq m$ such that $\left\lfloor\frac{3 \sum_{s=1}^{j-1}(n+s)+2}{2}\right\rfloor \geq \mathrm{k}$

Let

$$
\begin{aligned}
& w=\min \left\{j ; \quad 2 \leq j \leq m \text { such that }\left\lfloor\frac{3 \sum_{s=1}^{j-1}(n+s)+2}{2}\right\rfloor \geq \mathrm{k}\right\} \\
& \psi_{2}\left(x_{i}^{j} y_{i}^{j}\right)=\left\{\begin{array}{lll}
3 \sum_{s=1}^{w-1}(n+s)-2 k+i, & \text { if } & j=w \\
3 \sum_{s=1}^{w-1}(n+s)-2 k+i+3 n+3 j-1, & \text { if } & w+1 \leq j \leq m
\end{array}\right.
\end{aligned}
$$

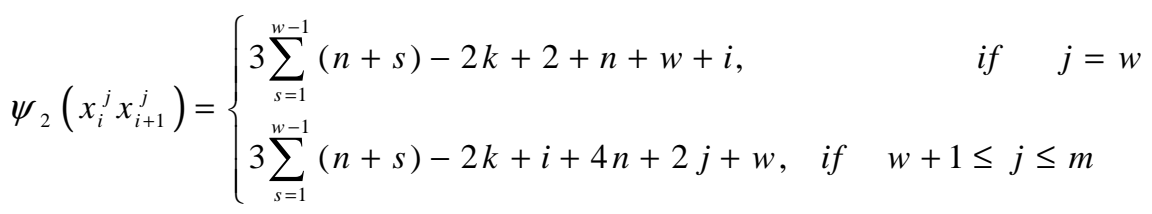

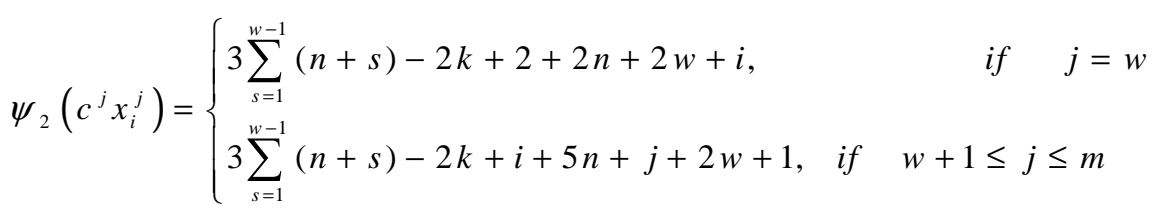

Under the labeling $\psi_{2}$ the total weights of the edges are described as follows:

(i) The edges $x_{i}^{1} y_{i}^{1}, x_{i}^{1} x_{i+1}^{1}$ and $c^{1} x_{i}^{1}$ for $1 \leq i \leq n+1$ receive consecutive integers from the interval $[3,3+n],[4+n, 2 n+4]$ and $[2 n+5,3 n+5]$, respectively.

(ii) The edges $x_{i}^{j} y_{i}^{j}$ for $1 \leq i \leq n+j, 2 \leq j \leq m$ receive consecutive integers from the interval $\left[3 \sum_{s=1}^{j-1}(n+s)+3,3 \sum_{s=1}^{j-1}(n+s)+n+j+2\right]$,

(iii) The edges $x_{i}^{j} x_{i+1}^{j}$ for $1 \leq i \leq n+j, 2 \leq j \leq m$ receive consecutive integers from the interval $\left[3 \sum_{s=1}^{j-1}(n+s)+n+j+3,3 \sum_{s=1}^{j-1}(n+s)+2 n+2 j+2\right]$, 
(iv) The edges $c^{j} x_{i}^{j}$ for $1 \leq i \leq n+j, 2 \leq j \leq m$ receive consecutive integers from the interval $\left[3 \sum_{s=1}^{j-1}(n+s)+2 n+2 j+3,3 \sum_{s=1}^{j}(n+s)+2\right]$,

It is not difficult to see that all vertex and edge labels are at most $k$ and the edgeweights of the edges $c^{j} x_{i}^{j}, x_{i}^{j} y_{i}^{j}$ and $x_{i}^{j} x_{i+1}^{j}$ are pairwise distinct. Thus, the resulting labeling is a total edge irregular $k$-labeling. This concludes the proof.

\section{Conclusion}

In this paper, we have determined the exact value of the total edge irregularity strength of the disjoint union of $m$ copies of a helm graph as well as the disjoint union of non-isomorphic helm graphs $\bigcup_{j=1}^{m} H_{n+j}$. We conclude by stating the following open problem:

Open Problem. For $m \geq 2$ find the exact value of the total edge irregularity strength of a disjoint union of $m$ arbitrary helm graphs.

\section{Acknowledgements}

The authors wish to thank the anonymous referee for his/her valuable comments.

\section{References}

[1] Baca, M., Jendrol, S., Miller, M. \& Ryan, J., On Irregular Total Labellings, Discrete Math., 307, pp. 1378-1388, 2007.

[2] Chartrand, G., Jacobson, M.S., Lehel, J., Oellermann, O.R., Ruiz, S. \& Saba, F., Irregular Networks, Congr. Numer., 64, pp. 187-192, 1988.

[3] Bohman, T. \& Kravitz, D., On the Irregularity Strength of Trees, J. Graph Theory, 45, pp. 241-254, 2004.

[4] Frieze, A., Gould, R.J., Karonski, M. \& Pfender, F., On Graph Irregularity Strength, J. Graph Theory, 41, pp. 120-137, 2002.

[5] Jendrol, S., Tkac, M., \& Tuza, Z., The Irregularity Strength and Cost of The Union of Cliques, Discrete Math., 150, pp. 179-186, 1996.

[6] Nierhoff, T., A Tight Bound on The Irregularity Strength of Graphs, SIAM J. Discrete Math., 13, pp. 313-323, 2000.

[7] Karonski, M., Luczak, T. \& Thomason, A., Edge Weights and Vertex Colours, J. Combin. Theory B, 91, pp. 151-157, 2004.

[8] Ivanco, J. \& Jendrol, S., Total Edge Irregularity Strength of Trees, Discussiones Math. Graph Theory, 26, pp. 449-456, 2006. 
[9] Jendrol, S., Miskuf, J. \& Sotak, R., Total Edge Irregularity Strength of Complete and Complete Bipartite Graphs, Electron. Notes Discrete Math., 28, pp. 281-285, 2007.

[10] Jendrol, S., Miskuf, J. \& Sotak, R., Total Edge Irregularity Strength of Complete Graphs and Complete Bipartite Graphs, Discrete Math., 310, pp. 400-407, 2010.

[11] Miskuf, J. \& Jendrol, S., On Total Edge Irregularity Strength of The Grids, Tatra Mt. Math. Publ., 36, pp. 147-151, 2007.

[12] Nurdin, Salman, A.N.M. \& Baskoro, E.T., The Total Edge-Irregular Strengths of The Corona Product of Paths with Some Graphs, J. Combin., Math. Combin. Comput., 65, pp. 163-175, 2008.

[13] Brandt, S., Miskuf, J. \& Rautenbach, D., On A Conjecture about Edge Irregular Total Labellings, J. Graph Theory, 57, pp. 333-343, 2008.

[14] Al-Mushayt, O., Ahmad, A. \& Siddiqui, M.K., On the Total Edge Irregularity Strength of Hexagonal Grid Graphs, Australas. J. Combin. 53, pp. 263-271, 2012.

[15] Ahmad, A., Siddiqui, M.K. \& Afzal, D., On the Total Edge Irregularity Strength of Zigzag Graphs, Australasian. J. Combin., 54, pp. 141-149, 2012.

[16] Ahmad, A. \& Baca, M., Total Edge Irregularity Strength of A Categorical Product of Two Paths, Ars. Combin, in press.

[17] Ahmad, A. \& Baca, M., Edge Irregular Total Labeling of Certain Family of Graphs, AKCE J. Graphs. Combin., 6(1), pp. 21-29, 2009.

[18] Siddiqui, M.K., On Total Edge Irregularity Strength of A Categorical Product of Cycle And Path, AKCE J. Graphs. Combin., 9(1), pp. 43-52, 2012.

[19] Siddiqui, M.K., On Tes of Subdivision of Star, Int. J. of Math and Soft Compu., 2(1), pp. 75-82, 2012.

[20] Ahmad, A., Baca, M. \& Siddiqui, M.K., On Edge Irregular Total Labeling of Categorical Product of Two Cycles, Theory of Computing systems, doi: 10.1007/s00224-013-9470-3.

[21] Ahmad, A., Baca, M., Bashir, Y. \& Siddiqui, M.K., Total Edge Irregularity Strength of Strong Product of Two Paths, Ars Combin., 106, pp. 449-459, 2012.

[22] Ahmad, A., Baca, M. \& Numan, M., On Irregularity Strength of Disjoint Union of Friendship Graphs, Electronic J. Graph Theory and Appl., in press (accepted on 19 November 2012). 